\title{
A finite element model of stress-mediated vascular adaptation: application to abdominal aortic aneurysms
}

\author{
Shahrokh Zeinali-Davarani, Azadeh Sheidaei and Seungik Baek* \\ Department of Mechanical Engineering, Michigan State University, East Lansing, MI 48824-1224, USA
}

(Received 9 October 2009; final version received 19 May 2010)

\begin{abstract}
Despite rapid expansion of our knowledge of vascular adaptation, developing patient-specific models of diseased arteries is still an open problem. In this study, we extend existing finite element models of stress-mediated growth and remodelling of arteries to incorporate a medical image-based geometry of a healthy aorta and, then, simulate abdominal aortic aneurysm. Degradation of elastin initiates a local dilatation of the aorta while stress-mediated turnover of collagen and smooth muscle compensates the loss of elastin. Stress distributions and expansion rates during the aneurysm growth are studied for multiple spatial distribution functions of elastin degradation and kinetic parameters. Temporal variations of the degradation function are also investigated with either direct time-dependent degradation or stretch-induced degradation as possible biochemical and biomechanical mechanisms for elastin degradation. The results show that this computational model has the capability to capture the complexities of aneurysm progression due to variations of geometry, extent of damage and stress-mediated turnover as a step towards patient-specific modelling.
\end{abstract}

Keywords: arterial growth and remodelling; computational vascular mechanics; modelling cardiovascular diseases; patient-specific modelling

\section{Introduction}

Vascular tissue exhibits a remarkable ability to adapt in various physiological and pathological conditions, often thought to be governed by mechanical factors (Mulvany 1992; Driss et al. 1997; Jackson et al. 2005). During the past decades, it has been shown that theoretical modelling of vascular growth and remodelling (G\&R) can sharpen our understanding of roles of mechanical stimuli on such adaptations by testing multiple hypotheses based on the accumulated information from experimental studies in vascular pathophysiology and various clinical observations (Humphrey et al. 2009). Recently, these theoretical models have been incorporated within the finite element method (FEM) framework by many researchers (Watton et al. 2004; Menzel 2005; Baek et al. 2006; Hariton et al. 2007; Kroon and Holzapfel 2007, 2009; Kuhl et al. 2007; Figueroa et al. 2009; Watton and Hill 2009), with results showing great potential for computational G\&R simulations to become essential in patient-specific risk assessment of vascular diseases and their treatment.

FEM-based vascular G\&R simulations typically utilise microstructural information of structural components (collagen, elastin and smooth muscle (SM)) to define the mechanical state at a given time (either stress or strain), which is iteratively fed back for calculating the evolution of microstructural properties of the components due to mechano-sensitive cellular activities. Humphrey and cow- orkers presented FEM models of stress-mediated G\&R using a constrained mixture approach and modelled intracranial aneurysms (Baek et al. 2005; Baek et al. 2006; Figueroa et al. 2009). Hariton et al. (2007) studied the stress-driven collagen fibres remodelling in a human carotid bifurcation. On the other hand, strain-mediated models of vascular adaptation have also been developed and implemented to model cerebral and aortic aneurysms (Watton et al. 2004; Kroon and Holzapfel 2007, 2009; Watton and Hill 2009). Driessen et al. (2004), (2008) investigated stretch-mediated remodelling of collagen fibres in arterial tissues.

Elastin degradation and collagen turnover are main biomechanical processes in the enlargement of aneurysms. Abdominal aortic aneurysms (AAAs) have been associated with a decrease in elastin content (Rizzo et al. 1989; He and Roach 1994; Ghorpade and Baxter 1996; Powell 2002) resulting from proteolytic activity. It has also been suggested that stress (strain)-mediated collagen turnover plays an important role in aneurysm enlargement and it may differ among individuals depending on physiological and pathological conditions (Choke et al. 2005). Hence, both elastin degeneration and collagen turnover should be considered in the computational models of AAAs development. Watton et al. (2004) and Watton and Hill (2009) were the first to develop a mathematical model of the evolution of an AAA by accounting for elastin degradation and collagen turnover. In their study, spatial

*Corresponding author. Email: sbaek@egr.msu.edu

ISSN 1025-5842 print/ISSN 1476-8259 online

(C) 2011 Taylor \& Francis

DOI: $10.1080 / 10255842.2010 .495344$

http://www.informaworld.com 
and temporal alterations of elastin concentration were prescribed and collagen remodelling acted to maintain the strain in fibres to an equilibrium value.

Previous modelling of evolving vascular diseases have mainly focused on hypothesis testing with simple geometries; however, there is a pressing need to account for patient-specific anatomical and physiological information in FEM simulations of vascular diseases (Taylor and Humphrey 2009). In this work, we extend the previous FEM model of stress-mediated G\&R developed by Baek et al. (2005), (2006) to incorporate a non-axisymmetric geometry obtained from medical images. The computational method is, then, applied to model an enlarging AAA assuming that an AAA grows by elastin degradation/damage and stress-mediated collagen turnover governed by the local state of intramural stress. We contrast stress distributions and expansion rates of the computationally grown AAAs for multiple kinetic parameters and different spatial distribution of elastin damage.

Towards this end, we employ a theoretical model of arterial G\&R based on a constrained mixture approach and use a nonlinear FEM with linear triangular elements.

\section{Theoretical modelling of arterial G\&R}

\subsection{Kinematics and important configurations}

There are two very different timescales in arterial mechanics: a short-term timescale associated with pulsatile motion during the cardiac cycle (seconds) and a long-term timescale for G\&R (days to weeks or months). Following Figueroa et al. (2009), $t$ denotes time associated with the cardiac cycle, during which we assume no G\&R, and $s$ denotes time during G\&R. The configuration $\kappa_{t}$ is the current configuration and $\mathbf{x}(t)$ denotes the position of a particle in $\kappa_{t}$. Here, we make a tacit assumption that there exists a stress-free configuration $\kappa_{s f}$ of the vessel wall which is fixed at a given $s$. The vector $\mathbf{X}_{s f}(s)$ denotes the position vector of the particle in the stress-free configuration and $\mathbf{F}_{s f}(t)$ denotes the deformation gradient corresponding to the mapping from $\mathbf{X}_{s f}(s)$ to $\mathbf{x}(t)$. In arterial mechanics, the effect of the inertial forces in arterial wall motion is negligible (Humphrey and $\mathrm{Na} 2002$ ) and we define the current (in vivo) configuration $\kappa_{s}$ in the G\&R timescale as the configuration $\kappa_{t}$ under the mean pressure during the cardiac cycle.

For modelling arterial G\&R, it appears to be advantageous to introduce additional configurations shown in Figure 1 (Baek et al. 2006; Figueroa et al. 2009). The configurations $\kappa_{\tau}$ trace the in vivo configurations of body through time $\tau \in[0, s]$. Particularly, the configuration $\kappa_{0}$ represents a loaded in vivo configuration of a healthy artery. We assume that each constituent is pre-stretched when deposited into the tissue at time $\tau$ and the tensor $\mathbf{G}^{i}(\tau)$ represents the deposition stretch of constituent $i$. For computational purposes, we introduce a configuration $\kappa_{R}$

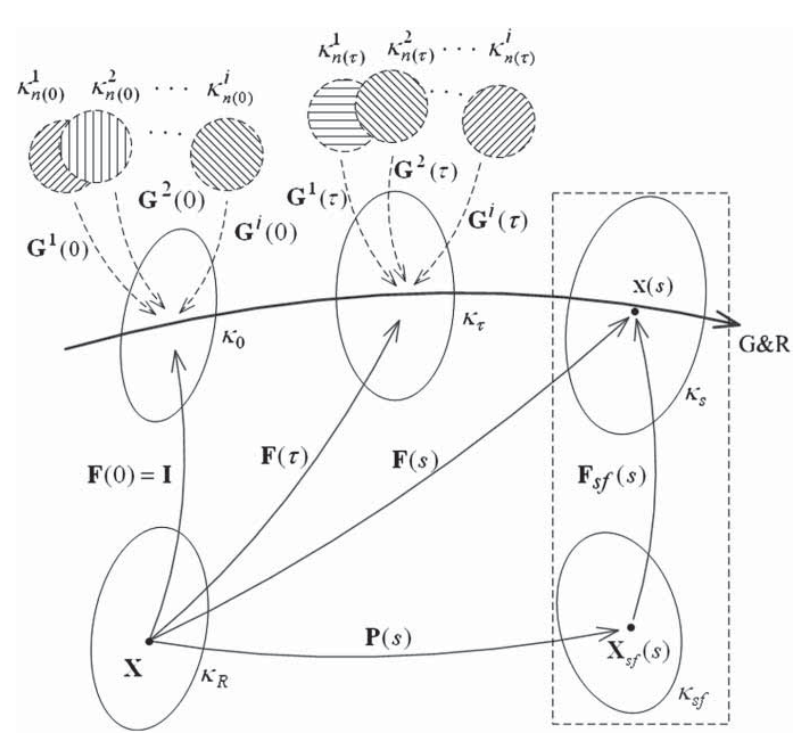

Figure 1. Schematic view of configurations involved in the G\&R simulation. A fixed reference configuration is considered for the computation of deformation associated with G\&R time $\tau=[0, s]$. It is chosen to coincide with the configuration of a healthy in vivo artery at time $\tau=0$ (i.e. $\kappa_{0}$ ). We imagine the existence of a stress-free configuration, $\kappa_{s f}$, associated with the current configuration $\kappa_{s}$.

called the (computational) reference configuration, that is, a fixed configuration. Although the configuration $\kappa_{R}$ is fixed in space, we assume that particles can be created or removed so that there is one-to-one mapping between $\kappa_{R}$ and $\kappa_{S}$ at time $s$. This assumption also implies that the total mass in $\kappa_{R}$ changes with time and always becomes the same as the total mass in $\kappa_{s}$. The position of a given particle in $\kappa_{R}$ is denoted by $\mathbf{X}$ and the deformation gradient $\mathbf{F}(s)$ is given corresponding to the mapping from $\kappa_{R}$ to $\kappa_{s}$. The stressfree configuration $\kappa_{s f}$ evolves in the G\&R time scale and $\mathbf{P}$ is the tensor representing its evolution. Because the total mass is preserved in the mapping from $\kappa_{R}$ to $\kappa_{s}$ at time $s$, the density with respect to the reference configuration, $\rho_{R}(s)$, can be calculated by

$$
\rho_{R}(s)=J(s) \rho(s),
$$

where $J(s)=\operatorname{det}[\mathbf{F}(s)]$ and $\rho(s)$ is the mass density in the current configuration at time $s$. Let $\varphi^{i}(s)$ denote the mass fraction of constituent $i$, i.e. $\sum_{i} \varphi^{i}(s)=1$. The mass density of constituent $i$ with respect to the reference configuration, $\rho_{R}^{i}(s)$, is defined as

$$
\rho_{R}^{i}(s)=\varphi^{i}(s) \rho_{R}(s) .
$$

The deformation gradient for each constituent $i$ at time $t$, relative to its natural configuration, $\mathbf{F}_{n(\tau)}^{i}(t)$, is given as Baek et al. (2006)

$$
\mathbf{F}_{n(\tau)}^{i}(t)=\mathbf{F}(t) \mathbf{F}^{-1}(\tau) \mathbf{G}^{i}(\tau),
$$


where $\mathbf{F}^{-1}(\tau) \mathbf{G}^{i}(\tau)$ is the tensor representing the prestretch of the constituent $i$ that has been produced at $\tau$ with respect to the reference configuration (see Figure 1). The deformation gradient $\mathbf{F}_{n(\tau)}^{i}(t)$ can also be written as

$$
\mathbf{F}_{n(\tau)}^{i}(t)=\mathbf{F}_{s f}(t) \mathbf{P}(s) \mathbf{F}^{-1}(\tau) \mathbf{G}^{i}(\tau),
$$

where, now, $\mathbf{P}(s) \mathbf{F}^{-1}(\tau) \mathbf{G}^{i}(\tau)$ represents the pre-stretch of the constituent $i$ that has been produced at $\tau$ with respect to $\kappa_{s f}$.

\subsection{Stress response and constitutive assumptions}

Consider an arterial wall tissue consisting of multiple structural components, e.g. elastin, multiple collagen families and SM. Following previous convention, we use the subscript ' $i$ ' to refer each constituent (e.g. elastin, collagen families and muscle) and the superscript ' $k$ ' to the $k$ th family of collagen fibres. Thus, $i=e, 1, \ldots, k, \ldots, m$ where $e$ denotes elastin and $m$ denotes SM. Although we adopt the G\&R formulation based on the constrained mixture approach (Humphrey and Rajagopal 2002), following its later modification (Baek et al. 2006; Figueroa et al. 2009), we take the point of view of a biological tissue comprising multiple constituents as a single continuum, where the mass fraction of each constituent and its prestretch are considered as attributes for a particle of the biological tissue. We further assume that for a given time $s$, the mechanical behaviour of the artery can be characterised by a hyperelastic model and we solve inflation problems with the principle of virtual work. Hence, let the Cauchy stress be given by Truesdell and Noll (1965),

$$
\mathbf{t}(t)=2 \rho(t) \mathbf{F}_{s f}(t) \frac{\partial \Psi\left(\mathbf{C}_{s f}(t)\right)}{\partial \mathbf{C}_{s f}(t)} \mathbf{F}_{s f}^{T}(t)
$$

where $\mathbf{C}_{s f}(t)=\mathbf{F}_{s f}^{T}(t) \mathbf{F}_{s f}(t)$ and $\Psi\left(\mathbf{C}_{s f}(t)\right)$ are the storedenergy function (per unit mass).

Since the stress-free configurations $\kappa_{s f}$ change with $\mathrm{G} \& \mathrm{R}$, we utilise a fixed computational reference configuration $\kappa_{R}$. Thus, (5) can be rewritten with respect to $\kappa_{R}$, using $\mathbf{F}(t)=\mathbf{F}_{s f}(t) \mathbf{P}(s),(1)$, and the chain rule, as

$$
\mathbf{t}(t)=\frac{2}{J(t)} \mathbf{F}(t) \frac{\partial\left\{\rho_{R}(s) \hat{\Psi}(\mathbf{C}(t))\right\}}{\partial \mathbf{C}(t)} \mathbf{F}^{T}(t)
$$

where

$$
\hat{\Psi}(\mathbf{C}(t))=\hat{\Psi}\left(\mathbf{P}^{T}(s) \mathbf{C}_{s f}(t) \mathbf{P}(s)\right)=\Psi\left(\mathbf{C}_{s f}(t)\right) .
$$

For a membrane model, using $J=J_{2 D} h / h_{R}$ for the thickness $h$, the membrane stress $\mathbf{T}$ can be given as

$$
\mathbf{T}(t)=\frac{2}{J_{2 D}(t)} \mathbf{F}_{2 D}(t) \frac{\partial M_{R}(s) \tilde{\Psi}\left(\mathbf{C}_{2 D}(t)\right)}{\partial \mathbf{C}_{2 D}(t)} \mathbf{F}_{2 D}^{T}(t)
$$

where the areal density $M_{R}(s)=h_{R} \rho_{R}(s)$ and $\tilde{\Psi}\left(\mathbf{C}_{2 D}(t)\right)=$ $\hat{\Psi}(\mathbf{C}(t))$ (e.g. see Holzapfel et al. (2000) for twodimensional formulation). For simplicity, we omit the subscript '2D' and define $w_{R}(s)=M_{R}(s) \tilde{\Psi}(\mathbf{C}(t))$. Then, (6) becomes

$$
\mathbf{T}(t)=\frac{2}{J(t)} \mathbf{F}(t) \frac{\partial w_{R}(\mathbf{C}(t))}{\partial \mathbf{C}(t)} \mathbf{F}^{T}(t) .
$$

To simulate arterial G\&R, we employ the stored energy equation $w_{R}(t ; s)$, the stored energy (per unit area) due to the deformation of $\mathbf{x}(t)$ at a given G\&R time $s$, as given by Figueroa et al. (2009)

$$
\begin{aligned}
w_{R}(t ; s)= & \sum_{i}\left\{M_{R}^{i}(0) Q^{i}(s) \Psi^{i}\left(\mathbf{C}_{n(0)}^{i}(t)\right)\right. \\
& \left.+\int_{0}^{s} m_{R}^{i}(\tau) q^{i}(s, \tau) \Psi^{i}\left(\mathbf{C}_{n(\tau)}^{i}(t)\right) \mathrm{d} \tau\right\},
\end{aligned}
$$

where $\Psi^{i}\left(\mathbf{C}_{n(\tau)}^{i}(t)\right)$ is the stored energy of constituent $i$ that has been produced, $\mathbf{C}_{n(\tau)}^{i}(t)=\left[\mathbf{F}_{n(\tau)}^{i}(t)\right]^{T} \mathbf{F}_{n(\tau)}^{i}(t), Q^{i}(s)$ is the fraction of the constituent $i$ that was present at time 0 and still remains at time $s$ (i.e. has not yet been removed), $m_{R}^{i}(\tau)$ is the mass production rate of the constituent $i$ at time $\tau$ per unit reference area and $q^{i}(s, \tau)$ is its survival function, that is, the fraction produced at time $\tau$ that remains at time $s$.

We employ constitutive relations for deposition tensors and stored energy functions for constituents used by Baek et al. (2006), (2007) and Figueroa et al. (2009). The mechanical property and the 'deposition stretch', named $G_{h}^{c}$, of the newly synthesised collagen fibres are assumed to be always the same. Let $\mathbf{m}^{k}(\tau)$ be the unit vector in the direction of the $k$ th collagen fibre produced at time $\tau$. The angle between $\mathbf{m}^{k}(\tau)$ and the first principal direction at time $\tau$ is denoted by $\alpha^{k}(\tau)$. We can find a unit vector $\mathbf{M}^{k}(\tau)$ in the reference configuration that corresponds to $\mathbf{m}^{k}(\tau)$, i.e.

$$
\mathbf{M}^{k}(\tau)=\frac{\mathbf{F}^{-1}(\tau) \mathbf{m}^{k}(\tau)}{\left|\mathbf{F}^{-1}(\tau) \mathbf{m}^{k}(\tau)\right|} .
$$

If the angle between $\mathbf{M}^{k}(\tau)$ and the first axis of the coordinate system is denoted by $\alpha_{R}^{k}(\tau)$, the stretch (of the fibre produced at time $\tau$ ) in the fibre direction from the reference to the current configuration is given by

$$
\lambda^{k}(t)=\sqrt{\mathbf{F}(t) \mathbf{M}^{k}(\tau) \cdot \mathbf{F}(t) \mathbf{M}^{k}(\tau)} .
$$

The stretch of the $k$ th fibre family from its natural to the current configuration can be, then, calculated as given by Baek et al. (2006)

$$
\lambda_{n(\tau)}^{k}(t)=\mathrm{G}_{h}^{c} \frac{\lambda^{k}(t)}{\lambda^{k}(\tau)},
$$


where $\lambda^{k}(\tau)$ is the stretch of the unit vector in the $k$ th fibre direction from the reference configuration to the configuration at time $\tau$. A similar approach can be adopted for SMs that are oriented primarily in the circumferential direction. Thus, the stretch of SM is given by $\lambda_{n(\tau)}^{m}(t)=\mathrm{G}_{h}^{m} \lambda_{2}(t) / \lambda_{2}(\tau)$, where $\mathrm{G}_{h}^{m}$ is the homeostatic stretch for the SM. The main structure of cross-linked elastin is formed at early stages of development, thus it is difficult to trace its production time and to specify $\mathbf{F}^{-1}(\tau)$ in (3). So we define a new tensor $\tilde{\mathbf{G}}^{e}=\mathbf{F}^{-1}(\tau) \mathbf{G}_{h}^{e}$, which represents a mapping from the natural configuration of elastin to the computational reference configuration. Then, $\mathbf{F}_{n}^{e}=\mathbf{F}(t) \tilde{\mathbf{G}}^{e}$ and $\tilde{\mathbf{G}}^{e}$ is postulated as $\tilde{\mathbf{G}}^{e}=$ $\operatorname{diag}\left\{G_{1}^{e}, G_{2}^{e}, \frac{1}{G_{1}^{e} G_{2}^{e}}\right\}$.

The stored energy functions for the elastin-dominated amorphous, collagen fibre families $\left(\Psi^{k}=\Psi^{c}\right)$ and passive $\mathrm{SM}$ are given as

$$
\begin{gathered}
\Psi^{e}\left(\mathbf{C}_{n}^{e}(t)\right)=\frac{c_{1}}{2}\left(C_{n[11]}^{e}+C_{n[22]}^{e}\right. \\
\left.+\frac{1}{C_{n[11]}^{e} C_{n[22]}^{e}-C_{n[12]}^{e}{ }^{2}}-3\right), \\
\Psi^{c}\left(\lambda_{n(\tau)}^{k}(t)\right)=\frac{c_{2}}{4 c_{3}}\left\{\exp \left[c_{3}\left(\lambda_{n(\tau)}^{k}{ }^{2}(t)-1\right)^{2}\right]-1\right\}, \\
\Psi^{m}\left(\lambda_{n(\tau)}^{m}(t)\right)= \\
\frac{c_{4}}{4 c_{5}}\left\{\exp \left[c_{5}\left(\lambda_{n(\tau)}^{m}{ }^{2}(t)-1\right)^{2}\right]-1\right\},
\end{gathered}
$$

where $C_{n[11]}^{e}, C_{n[22]}^{e}$ and $C_{n[12]}^{e}$ are components of $\mathbf{C}_{n}^{e}=\left[\mathbf{F}_{n}^{e}\right]^{T} \mathbf{F}_{n}^{e}$. Although the constitutive form is the same for collagen fibre families and SM, SM is much more compliant than collagen fibre families and has less contribution to the passive mechanical behaviour of the wall (Burton 1954). We use a potential function for the active tone of vascular SM as given by Zulliger et al. 2004, Baek et al. 2007

$$
\Psi_{\mathrm{act}}^{m}(t)=\frac{S}{\rho}\left\{\lambda_{2}(t)+\frac{1}{3} \frac{\left(\lambda_{M}-\lambda_{2}(t)\right)^{3}}{\left(\lambda_{M}-\lambda_{\mathrm{o}}\right)^{2}}\right\}
$$

where $\lambda_{M}$ and $\lambda_{0}$ are stretches at which the active force generation is maximum and zero, $\lambda_{2}$ is the stretch in circumferential direction at time $t$ and $S$ is the stress at the maximum contraction. Then, the total membrane strain energy becomes $w_{R}=w_{R(\text { passive })}+M_{R}^{m}(t) \Psi_{\text {act }}^{m}$.

\subsection{Stress-mediated $G \& R$}

In arteries, constituents can be continuously produced and removed and normal tissue maintains its mass and configuration by balanced turnover of constituents. The rates of production and removal change from their balanced normal (basal) values in response to changes in mechanical environment. Here, we assume that $m_{R}^{e}(s)=0$ and the rates of mass production for collagen fibres and SM are functions of a scalar measure of intramural stress, given by Baek et al. (2006)

$$
\begin{gathered}
m_{R}^{k}(s)=\frac{M_{R}^{c}(s)}{M_{R}^{c}(0)}\left(K_{g}^{k}\left(\sigma^{k}(s)-\sigma_{h}^{c}\right)+m_{\text {basal }}^{k}\right), \\
m_{R}^{m}(s)=\frac{M_{R}^{m}(s)}{M_{R}^{m}(0)}\left(K_{g}^{m}\left(\sigma^{m}(s)-\sigma_{h}^{m}\right)+m_{\text {basal }}^{m}\right),
\end{gathered}
$$

where $M_{R}^{c}(0)$ and $M_{R}^{m}(0)$ are the mass of collagen and SM per reference area of a healthy artery at time 0 , respectively. $K_{g}^{i}(i=1,2, \ldots, k, \ldots, m)$ is a scalar parameter that controls the stress-mediated growth, $m_{\text {basal }}^{i}$ is a basal rate of mass production for the constituent $i$ and

$$
\sigma^{k}(s)=\frac{\left\|\mathbf{T}^{c}(s) \mathbf{m}^{k}(s)\right\|}{h^{c}(s)}, \quad \sigma^{m}(s)=\frac{\left\|\mathbf{T}^{m}(s) \mathbf{m}^{m}(s)\right\|}{h^{m}(s)},
$$

where $\mathbf{T}^{c}(s)$ and $\mathbf{T}^{m}(s)$ are the Cauchy membrane stress contributed by collagen and SM at time $s$ (i.e. $\left.\mathbf{T}^{c}(s)=\sum_{k} \mathbf{T}^{k}(s)\right) . h^{c}(s)$ and $h^{m}(s)$ are contributions of collagen and blue SM to the total thickness at time $s$. Also, let

$$
q^{i}(s, \tau)=\left\{\begin{array}{ll}
\exp \left(-\int_{\tau}^{s} k_{q}^{i}(\tilde{\tau}) \mathrm{d} \tilde{\tau}\right) & s-\tau \leq a_{\max }^{i} \\
0 & s-\tau>a_{\max }^{i}
\end{array}\right\},
$$

where $k_{q}^{i}(\tilde{\tau})$ can be a function of circumferential stress, wall shear stress or other state variables. $a_{\max }^{i}$ is the maximum life span of the constituent $i$.

The new collagen is deposited with a preferred alignment. Following Baek et al. (2006), we assume that the alignment of the newly produced collagen is influenced by the orientation of the existing collagen and it consequently aligns along the direction of the existing collagen family.

\section{Computational considerations}

\subsection{Local Cartesian coordinate system}

We assume that the wall is a thin membrane, with $\mathbf{X}=$ $\left\{X_{1}, X_{2}, X_{3}\right\}$ and $\mathbf{x}=\left\{x_{1}, x_{2}, x_{3}\right\}$ being the reference and current positions in the global Cartesian coordinate system with base vectors $\left\{\mathbf{E}_{1}, \mathbf{E}_{2}, \mathbf{E}_{3}\right\}$ (Figure 2). We use linear triangular elements for developing a nonlinear FEM model of a non-axisymmetric cylindrical membrane and define a local Cartesian coordinate system for each element in order to facilitate calculation of the local deformation 


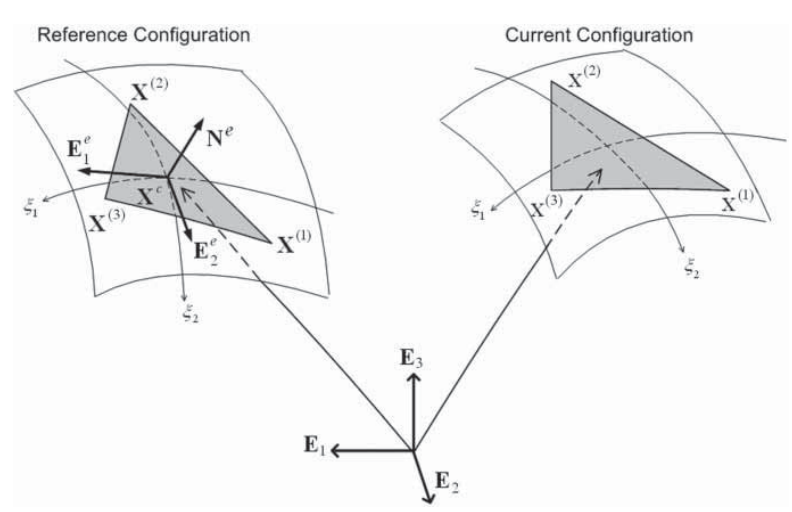

Figure 2. Orthonormal base vectors for a global Cartesian coordinate system are $\left\{\mathbf{E}_{1}, \mathbf{E}_{2}, \mathbf{E}_{3}\right\}$. Those for a local Cartesian coordinate system are $\mathbf{E}_{1}^{e}, \mathbf{E}_{2}^{e}$ (associated with $\xi_{1}, \xi_{2}$ ), and $\mathbf{E}_{3}^{e}=\mathbf{N}^{e}$ at a point set to be the centre point of a linear triangular element tangent to the membrane at $\mathbf{X}^{c} . \mathbf{E}_{1}^{e}$ and $\mathbf{E}_{2}^{e}$ are orthonormal bases on the tangent plane and $\mathbf{N}^{e}$ is the outward unit normal vector.

gradient and prescribe material anisotropy. The centroid of an element $\mathbf{X}^{c}=\left\{X_{1}^{c}, X_{2}^{c}, X_{3}^{c}\right\}$ becomes the origin of the local Cartesian coordinate system, and 'element-wise' orthogonal surface coordinates $\xi_{1}$ and $\xi_{2}$ are allocated to any point on the element with respect to the origin (Figure 2).

A linear triangular element has three nodal points $\left\{\mathbf{X}^{(1)}, \mathbf{X}^{(2)}, \mathbf{X}^{(3)}\right\}$ in the reference and $\left\{\mathbf{x}^{(1)}, \mathbf{x}^{(2)}, \mathbf{x}^{(3)}\right\}$ in the current configurations and $X_{i}^{c}=(1 / 3) \sum_{k} X_{i}^{(k)}$ $(i, k=1,2,3)$. Now, we introduce local bases $\mathbf{E}_{1}^{e}$ and $\mathbf{E}_{2}^{e}$ in the plane of a linear triangular element (i.e. tangent plane) and $\mathbf{E}_{3}^{e} \equiv \mathbf{N}^{e}$ the outward normal to that plane (Figure 2). The base vector $\mathbf{E}_{1}^{e}$ is set to a unit vector aligned towards the axial direction of a model of the artery (a unit tangent vector to $\xi_{1}$ at the element centre point). The outward normal vector $\mathbf{N}^{e}$ is readily obtained by cross product of vectors connecting nodal points. The base vector $\mathbf{E}_{2}^{e}$ is then calculated as $\mathbf{E}_{2}^{e}=\mathbf{N}^{e} \times \mathbf{E}_{1}^{e}$. If $\mathbf{X}^{e}=$ $\left\{X_{1}^{e}, X_{2}^{e}, X_{3}^{e}\right\}$ and $\mathbf{x}^{e}=\left\{x_{1}^{e}, x_{2}^{e}, x_{3}^{e}\right\}$ are the reference and current position vectors in the local Cartesian coordinate system, the two-dimensional right Cauchy-Green deformation tensor is calculated as given by Kyriacou et al. (1996), Park and Youn (1998)

$$
\mathbf{C}=\left(\frac{\partial x^{e}}{\partial \xi_{\alpha}} \cdot \frac{\partial x^{e}}{\partial \xi_{\beta}}\right) \mathbf{E}_{\alpha}^{e} \otimes \mathbf{E}_{\beta}^{e}
$$

where $\alpha, \beta=1,2$.

\subsection{Finite element formulation}

The weak form for the membrane can be obtained from the principle of virtual work (Kyriacou et al. 1996),

$$
\delta I=\int_{S} \delta w \mathrm{~d} A-\int_{S} P \mathbf{n} \cdot \delta x \mathrm{~d} a=0 .
$$

In an FEM model, we seek an approximate solution to (22). Let a finite approximation of the current position be

$$
\mathbf{x}=\boldsymbol{\Phi} \mathbf{x}^{p}, \quad x_{i}=\Phi_{i A} x_{A}^{p},
$$

where $\mathbf{x}^{p}$ and $\boldsymbol{\Phi}$ are the nodal vector for the current position and shape function matrix, respectively. Then, the governing equation for an element can be obtained from (22) as

$$
\{\mathcal{F}\}_{P}^{e}=\int_{S^{e}}\left(\frac{\partial w}{\partial C_{\alpha \beta}} \frac{\partial C_{\alpha \beta}}{\partial x_{P}^{p}}-\tilde{P}_{i} \Phi_{i P}\right) \mathrm{d} A=0
$$

where for a linear triangular element,

$$
\tilde{P}_{i}=P \frac{\epsilon_{i j k}\left(x_{j}^{(2)}-x_{j}^{(1)}\right)\left(x_{k}^{(3)}-x_{k}^{(1)}\right)}{\left\|\epsilon_{l m n}\left(X_{m}^{(2)}-X_{m}^{(1)}\right)\left(X_{n}^{(3)}-X_{n}^{(1)}\right)\right\|} .
$$

We use the Newton-Raphson method to solve (24), and the tangent matrix can be given by

$$
\begin{aligned}
{[\mathbf{K}]_{P Q}=} & {\left[\frac{\partial \mathcal{F}}{\partial x^{p}}\right]_{P Q}^{e}=\int_{S^{e}}\left(\frac{\partial^{2} w}{\partial C_{\alpha \beta} \partial C_{\gamma \omega}} \frac{\partial C_{\alpha \beta}}{\partial x_{P}^{p}} \frac{\partial C_{\gamma \omega}}{\partial x_{Q}^{p}}\right.} \\
& \left.+\frac{\partial w}{\partial C_{\alpha \beta}} \frac{\partial^{2} C_{\alpha \beta}}{\partial x_{P}^{p} \partial x_{Q}^{p}}-\Phi_{i P} \tilde{P}_{i, Q}\right) \mathrm{d} A .
\end{aligned}
$$

Note that $(i, j, k, l, m, n=1,2,3),(\alpha, \beta, \gamma, \omega=1,2)$, and $\left(A, B, M, P, Q=1,2,3, \ldots, n_{p}\right)$, where $n_{p}$ is the number of nodes in an element multiplied by 3, i.e. $n_{p}=9$.

Any point $\mathbf{X}=\left\{X_{1}, X_{2}, X_{3}\right\}$ in the global Cartesian coordinate system can be transformed to $\mathbf{X}^{e}=$ $\left\{X_{1}^{e}, X_{2}^{e}, X_{3}^{e}\right\}$ in the local coordinate system using

$$
X_{i}^{e}=\mathbf{E}_{i}^{e} \cdot\left(\mathbf{X}-\mathbf{X}^{c}\right)=Q_{i j}\left(X_{j}-X_{j}^{c}\right)
$$

where $Q_{i j}=\mathbf{E}_{i}^{e} \cdot \mathbf{E}_{j}$. It is convenient to define $9 \times 1$ vectors for the nodal points as

$$
\begin{aligned}
& \mathbf{X}^{e p}=\left\{X_{1}^{e(1)}, X_{2}^{e(1)}, X_{3}^{e(1)}, X_{1}^{e(2)}, X_{2}^{e(2)}, X_{3}^{e(2)},\right. \\
& \left.X_{1}^{e(3)}, X_{2}^{e(3)}, X_{3}^{e(3)}\right\}^{T} \\
& \mathbf{x}^{e p}=\left\{x_{1}^{e(1)}, x_{2}^{e(1)}, x_{3}^{e(1)}, x_{1}^{e(2)}, x_{2}^{e(2)}, x_{3}^{e(2)},\right. \\
& \left.x_{1}^{e(3)}, x_{2}^{e(3)}, x_{3}^{e(3)}\right\}^{T} \text {, }
\end{aligned}
$$

$$
\begin{aligned}
& \mathbf{X}^{p}=\left\{X_{1}^{(1)}, X_{2}^{(1)}, X_{3}^{(1)}, X_{1}^{(2)}, X_{2}^{(2)}, X_{3}^{(2)}, X_{1}^{(3)},\right. \\
& \left.X_{2}^{(3)}, X_{3}^{(3)}\right\}^{T} \text {, } \\
& \mathbf{x}^{p}=\left\{x_{1}^{(1)}, x_{2}^{(1)}, x_{3}^{(1)}, x_{1}^{(2)}, x_{2}^{(2)}, x_{3}^{(2)}, x_{1}^{(3)},\right. \\
& \left.x_{2}^{(3)}, x_{3}^{(3)}\right\}^{T} \text {. }
\end{aligned}
$$




$$
\mathbf{X}^{c}=\left\{X_{1}^{c}, X_{2}^{c}, X_{3}^{c}, X_{1}^{c}, X_{2}^{c}, X_{3}^{c}, X_{1}^{c}, X_{2}^{c}, X_{3}^{c}\right\}^{T} .
$$

The coordinate transformation between global and local coordinates can be expressed by

$$
\mathbf{X}^{e p}=\tilde{\boldsymbol{Q}}\left(\mathbf{X}^{p}-\mathbf{X}^{c}\right), \quad \mathbf{x}^{e p}=\tilde{\boldsymbol{Q}}\left(\mathbf{x}^{p}-\mathbf{X}^{c}\right),
$$

where a $9 \times 9$ matrix $\tilde{\mathbf{Q}}$ is given by

$$
\tilde{\mathbf{Q}}=\left[\begin{array}{lll}
\mathbf{Q} & \mathbf{0} & \mathbf{0} \\
\mathbf{0} & \mathbf{Q} & \mathbf{0} \\
\mathbf{0} & \mathbf{0} & \mathbf{Q}
\end{array}\right] \text {. }
$$

Note that the local position vectors of element nodes in the current configuration, $\mathbf{x}^{e p}$, are expressed with respect to the local coordinate system associated with that element in the reference configuration. The position vector in the local coordinate system is

$$
\mathbf{x}^{e}\left(\xi_{1}, \xi_{2}\right)=\boldsymbol{\Phi} \mathbf{x}^{e p}
$$

where

$$
\boldsymbol{\Phi}=\left[\begin{array}{ccccccccc}
\phi^{1} & 0 & 0 & \phi^{2} & 0 & 0 & \phi^{3} & 0 & 0 \\
0 & \phi^{1} & 0 & 0 & \phi^{2} & 0 & 0 & \phi^{3} & 0 \\
0 & 0 & \phi^{1} & 0 & 0 & \phi^{2} & 0 & 0 & \phi^{3}
\end{array}\right]
$$

and $\phi^{1}, \phi^{2}$ and $\phi^{3}$ are linear shape functions of $\xi_{1}$ and $\xi_{2}$ for the element. Using (33), (35) can be rewritten as

$$
\mathbf{x}^{e}=\boldsymbol{\Phi} \tilde{\mathbf{Q}}\left(\mathbf{x}^{p}-\mathbf{X}^{c}\right), \quad\left[x_{i}^{e}=\Phi_{i A} \tilde{Q}_{A B}\left(x_{B}^{p}-X_{B}^{c}\right)\right] .
$$

For convenience, let us define $\boldsymbol{\Phi}^{e}$ as

$$
\Phi_{i B}^{e}=\Phi_{i A} \tilde{Q}_{A B} .
$$

The components of the derivative of the local position vector with respect to the local coordinate are

$$
x_{i, \alpha}^{e}=\Phi_{i A, \alpha} \tilde{Q}_{A B} x_{B}^{p} \quad \text { or } \quad x_{i, \alpha}^{e}=\Phi_{i A, \alpha}^{e} x_{A}^{p} .
$$

Thus, components of the right Cauchy-Green tensor (21) can be obtained as

$$
C_{\alpha \beta}=x_{i, \alpha}^{e} x_{i, \beta}^{e}=\Phi_{i A, \alpha}^{e} x_{A}^{p} \Phi_{i M, \beta}^{e} x_{M}^{p},
$$

where the basis is $\mathbf{E}_{\alpha}^{e} \otimes \mathbf{E}_{\beta}^{e}$.

\subsection{Numerical solutions}

The spatial integration in (24) is approximated by using Gauss integration. At every Gauss point, the temporal integrations in (22) are calculated by using the trapezoidal rule (Press et al. 1992) over past times. Although the survival function (20) is given by an exponential function, we define a maximum lifetime $a_{\max }^{i}$ and truncate the value if $s-\tau \leq a_{\max }^{i}$. Thus, the numerical integration can be done over the time interval $\left[s-a_{\max }^{i}, s\right]$ using fixed number of discretisation points. Equations (17), (18) and (24) are solved iteratively for the nodal positions and rates of mass production at a given time $s$. Briefly, an initial guess of $m_{R}^{i}(s)$ is made at each Gauss point based on the previous time step, and (24) is solved for the current positions using the nonlinear FEM technique prescribed in the previous section. Then, $m_{R}^{i}(s)$ is updated using the FEM solution along with (17) and (18). In this way, rates of mass production and displacements at the current time $s$ are updated iteratively until solutions converge to the prescribed tolerance.

For testing the utility of the present work, we apply the computational model for simulation of an AAA. One Gauss point is used for the integration. It has been found that the nonlinear FEM analysis does not always converge well with (26). To remedy this, we modify the tangent matrix by

$$
\begin{aligned}
{[\mathbf{K}]_{P Q}=} & \zeta \int_{S^{e}}\left(\frac{\partial^{2} w}{\partial C_{\alpha \beta} \partial C_{\gamma \omega}} \frac{\partial C_{\alpha \beta}}{\partial x_{P}^{p}} \frac{\partial C_{\gamma \omega}}{\partial x_{Q}^{p}}+\frac{\partial w}{\partial C_{\alpha \beta}} \frac{\partial^{2} C_{\alpha \beta}}{\partial x_{P}^{p} \partial x_{Q}^{p}}\right) \mathrm{d} A \\
& -\int_{S^{e}} \Phi_{i P} \tilde{P}_{i, Q} \mathrm{~d} A,
\end{aligned}
$$

where $\zeta=2.0$ shows a good convergence for all simulations in this work.

\section{Simulation of an AAA growth}

\subsection{A geometric model and mesh generation}

A 3D computational geometry of a healthy aorta is reconstructed from magnetic resonance images of a healthy subject using Simvascular (Cardiovascular lab, Stanford University). The computational domain is extended to the upper part of abdominal aorta (proximal side) and iliac branches (distal side) for future use in haemodynamic simulations (Sheidaei et al. 2010). For simulation of the aneurysm, we use only the central region of the geometric model which is separately meshed with triangular elements (1584 elements) using Gambit (Lebanon, NH, USA).

\subsection{Initialisation for $G \& R$ simulation}

In normal physiological conditions, production and removal of each constituent are balanced such that the vessel maintains its shape under a preferred homeostatic state. For an idealised model of the blood vessel, the in vivo material properties are typically assumed to be constant 
Table 1. Constitutive and kinetic parameters for each constituents used in initialisation and G\&R simulations.

\begin{tabular}{ll}
\hline Elastin: & $c_{1}=112 \mathrm{~Pa} / \mathrm{kg}, G_{1}^{e}=1.25, G_{2}^{e}=1.25$ \\
Collagen: & $c_{2}=917 \mathrm{~Pa} / \mathrm{kg}, c_{3}=25, G_{h}^{c}=1.07, k_{q}^{c}=0.02$ \\
SM: & $c_{4}=27 \mathrm{~Pa} / \mathrm{kg}, c_{5}=8.5, G_{h}^{m}=1.2, k_{q}^{m}=0.02$, \\
& $S=50 \mathrm{kPa}, \lambda_{M}=1.2, \lambda_{0}=0.7$ \\
Others: & $\rho=1050 \mathrm{~kg} / \mathrm{m}^{3}, \sigma_{h}^{c}=\sigma_{h}^{m}=135 \mathrm{kPa}$ \\
\hline
\end{tabular}

over the domain with parameter values estimated from experimental data, while the homeostatic assumption is imposed as a constraint (Baek et al. 2007; Figueroa et al. 2009; Valentín et al. 2009). When medical image-based geometric models are used, however, it is not a trivial task to prescribe the distribution of material and geometric parameters while maintaining the homeostatic mechanical state under physiological pressure. For an approximation, we first prescribe material constitutive parameters shown in Table 1. Other parameters, $c_{1}, c_{2}$ and $c_{4}$, are calculated inversely assuming that all constituents have the same homeostatic stress value (i.e. $\sigma^{i}=\sigma_{h}$ ) in a healthy state. For that, four discrete fibre families are assumed to be initially aligned in 0, 90, 45 and -45 (axial, circumferential and helical directions, respectively), and constant volume fractions for all constituents are prescribed. Then, using strain energy functions (13)-(16), $c_{1}, c_{2}$, and $c_{4}$ and are obtained by

$$
\begin{gathered}
c_{1}=\sigma_{h}\left\{\rho\left(G_{1}^{e 2}-\left(G_{1}^{e} G_{2}^{e}\right)^{-2}\right)\right\}^{-1}, \\
c_{2}=\sigma_{h}\left\{\rho G_{h}^{c 2}\left(G_{h}^{c 2}-1\right) \exp \left\{c_{3}\left(G_{h}^{c 2}-1\right)^{2}\right\}\right. \\
\left.\sum_{k=1}^{4} \frac{M_{R}^{k}}{M_{R}^{c}} \sin ^{2} \alpha^{k}\right\}^{-1}, \\
c_{4}=\frac{\sigma_{h}-S\left\{1-\left(\frac{\lambda_{M}-1}{\lambda_{M}-\lambda_{0}}\right)^{2}\right\}}{\rho G_{h}^{m 2}\left(G_{h}^{m 2}-1\right) \exp \left\{c_{5}\left(G_{h}^{m 2}-1\right)^{2}\right\}} .
\end{gathered}
$$

Regional wall thickness is initially approximated by estimating the cross-sectional mean radius and using the Laplace equation, $\sigma=\operatorname{Pr} / h$. Then, using the G\&R simulation, we let the vessel wall adapt to an equilibrium state with a high value of stress-mediated parameters $\left(K_{g}^{k}=K_{g}^{m}=1\right)$. Deviation of stress in three families of collagen fibres (i.e. axial and helical directions) from the desired homeostatic stress $\left(\sigma^{k}-\sigma_{h}^{c}\right) / \sigma_{h}^{c}$ is plotted before and after 300 'days' of this initial simulation in Figure 3. Stress deviation of the fibre family along the circumferential direction was less than the other fibres (not shown). Figure 4(a) and (b), respectively, depicts the displacement
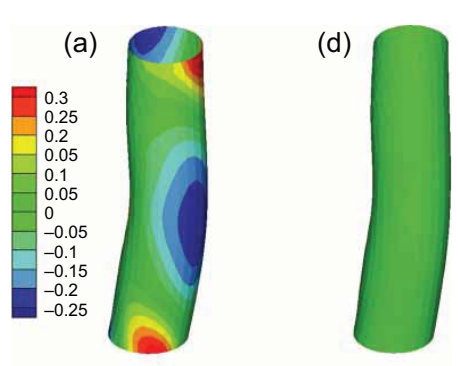

(b)
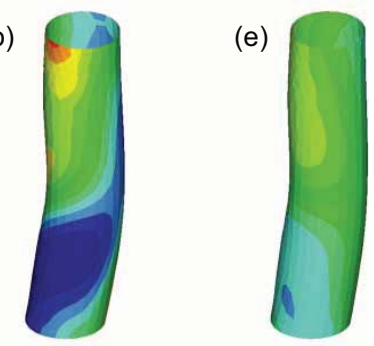

(c)

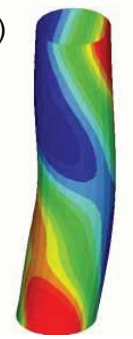

(f)

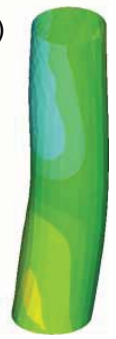

Figure 3. Deviation of the intramural stress from the homeostatic value $\left(\sigma^{k}-\sigma_{h}^{c}\right) / \sigma_{h}^{c}$ in axial and two helical fibre directions before (a, b and c) and after (d, e and f) 300 days of the initial simulation.

and the change in the geometry from the reference configuration after 300 days of this initial simulation. The stress deviation of each fibre family is reduced substantially after the initialisation process while causing little change in the geometry from its original/reference configuration (maximum displacement is about $1.5 \mathrm{~mm}$ ).

(a)

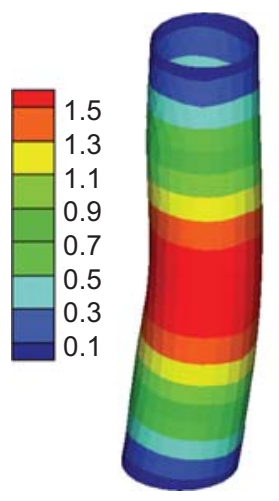

(b)

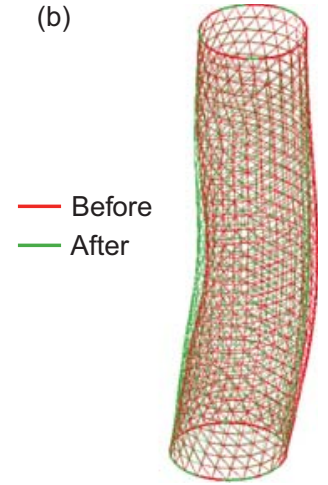

Figure 4. (a) Magnitude of the displacement ( $\mathrm{mm}$ ) calculated from the reference configuration after 300 days of the initial simulation (b) and the corresponding geometry before and after 300 days. 


\subsection{Simulation cases}

After initialisation, an AAA is initiated by introducing damage to the aorta, where elastin is degraded/removed from the blood vessel. The spatial and temporal function for elastin damage is given as

$$
D(\mathbf{X}, s)=\left(1-0.01^{s / T}\right) f(\mathbf{X})
$$

where $T$ is a time constant and $f(\mathbf{X})$ defines the spatial distribution of damage. $D(\mathbf{X}, s) \in[0,1]$ is the ratio of the degenerated elastin to the initial amount, where $D(\mathbf{X}, s)=1$ for complete degradation. First, we assume a limiting case when $T \rightarrow 0^{+}$such that $D(\mathbf{X}, s)$ becomes only a spatial function, $f(\mathbf{X})$. That is, the damage (elastin removal) is applied immediately after the initial simulation for a homeostatic condition, and the amount of damage is kept constant during G\&R. Four spatial functions are used for representing different cases of elastin damage. The areal density of elastin after applying different damages is plotted in Figure 5. Cases (1) and (2) correspond to damage shapes distributed to a relatively large area on the concave

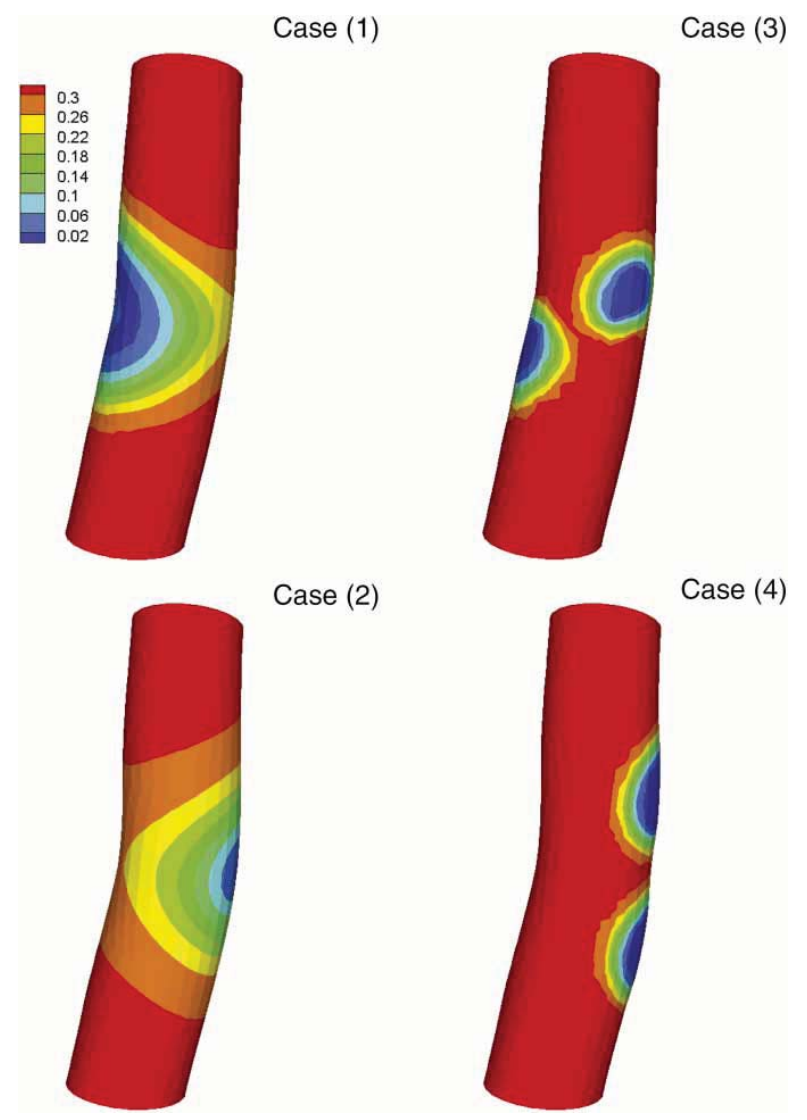

Figure 5. Areal mass density of elastin $\mathrm{kg} / \mathrm{m}^{2}$ for different simulation Cases (1)-(4). Cases (1) and (2) correspond to different damages shapes distributed to relatively large area which are applied at different locations (concave and convex sides) and Cases (3) and (4) correspond to more localised damage shapes, but at multiple locations. and convex sides, respectively. Cases (3) and (4) correspond to more localised damage shapes applied at multiple locations. Second, the 'time dependent' elastin degradation is investigated using (44) with a spatial function of Case (3) considering $T=10 \times 365$ (days). For final simulation, we assume 'stretch-induced' degradation, where additional removal of elastin occurs during aneurysm growth due to stretch-induced damage. Specifically, the function for elastin damage can be written as

$$
D(\mathbf{X}, s)=f(\mathbf{X})+g\left(I_{1}^{e}(\mathbf{X}, s)\right)[1-f(\mathbf{X})],
$$

where $I_{1}^{e}(\mathbf{X}, s)$ is the first invariant of the $\mathbf{C}_{n}^{e}$ and

$g\left(I_{1}^{e}\right)=\left\{\begin{array}{cc}1 & \left(I_{1}^{e}-3\right) \geq 4.48 \\ 1-\sin \left(\frac{\pi\left(7.48-I_{1}^{e}\right.}{2 \times 1.48}\right) & 3 \leq\left(I_{1}^{e}-3\right)<4.48 \\ 0 & \left(I_{1}^{e}-3\right)<3\end{array}\right\}$.

It is believed that elastin plays an important role in controlling SM cells migration/proliferation (Li et al. 1998; Karnik et al. 2003) as well as their phenotype modulation (Ailawadi et al. 2009) by stabilising extracellular matrix. The amount of SM in our model is changed proportionally with the initial elastin degradation which reduces both passive and active contributions of SM to the wall mechanical properties.

\subsection{Results}

Distribution of the maximum principal stress and the areal mass density of collagen at 50,1200 and 2700 days of G\&R for Case (3) are shown in Figure 6. The damage to elastin introduced at $s=0$ causes a sharp increase in stress at the location of the damage. Stress-mediated collagen production increases the areal density of collagen as the lesion enlarged, compensating the loss of elastin, and the value of the peak stress initially starts to decrease. As the aneurysm enlarges further, however, the stress level is shown to increase again (see Figure 6(c)). The shape of aneurysm and stress distributions resulted from Cases (1), (2) and (4) are plotted at 50 and 2700 days of the G\&R simulation in Figure 7 . The simulation suggests that the aneurysmal wall tends to enlarge more in the convex side of the vessel than in the concave side. For example, Figure 7(d) shows a large amount of dilatation in the convex side even with the initial damage introduced in the concave side. Different spacial functions for elastin damage result in a variety of shapes with different expansion rates, although in all cases the same kinetic parameter $K_{g}=0.05$ was used. Figure 8 plots expansion rates (maximum diameter increase per unit time) for simulations with four different cases. Cases (1) and (2) result in higher expansion rates compared to Cases (3) 

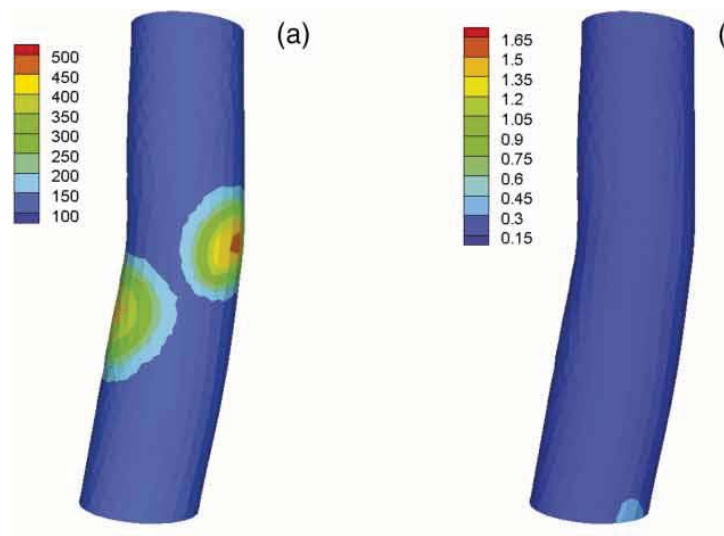

(d)

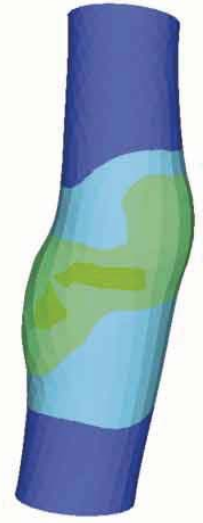

(b)

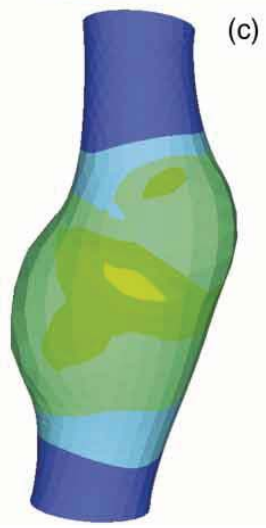

(c)
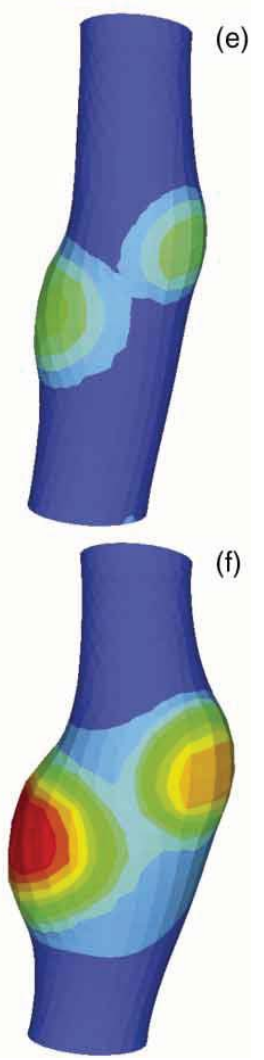

Figure 6. Maximum principal stress distribution $(\mathrm{kPa})$ for the simulation Case (3) at 50, 1200 and 2700 days after the initial damage ( $\mathrm{a}, \mathrm{b}$ and $\mathrm{c}$ ) and the corresponding areal mass density of collagen $(\mathrm{d}$, e and $\mathrm{f})\left(\mathrm{kg} / \mathrm{m}^{2}\right)$.

and (4), which might be due to the wider area of the damage prescribed for Cases (1) and (2). The effect of kinetic parameter $\left(K_{g}=0.02,0.05\right.$ and 0.1$)$ on the expansion rate of the aneurysm is shown for Case (2) in Figure 9. Apparently, simulation with $K_{g}=0.02$ results in an increase in the aneurysm expansion rate, simulation with $K_{g}=0.05$ causes a linear enlargement over time (constant expansion rate) and a higher value of the kinetic parameter $\left(K_{g}=0.1\right)$ even stabilises the aneurysm growth. Figure 10 shows the areal density of elastin and the maximum principal stress for the 'time dependent' case at 50 and

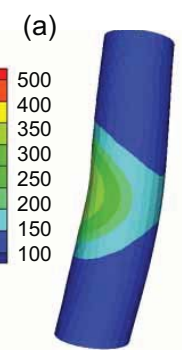

(b)

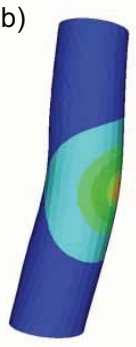

(c)

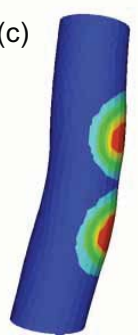

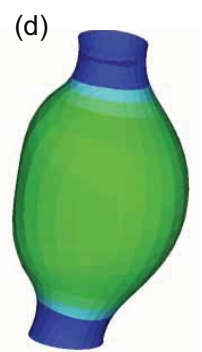

(e)

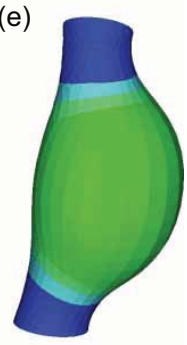

(f)

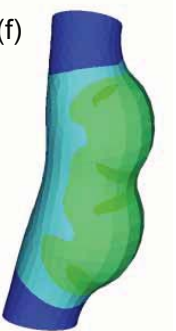

Figure 7. Maximum principal stress $(\mathrm{kPa})$ for simulation Cases (1), (2) and (4) after 50 (a, b and c) and 2700 (d, e and f) days.

2700 days of the G\&R simulation. At 50 days, changes in stress are small because of the small amount of elastin degradation (Figure 10(a)). The stress increases gradually as the amount of elastin degradation increases, and at 2700 days the maximum principal stress reaches a similar value as in Case (3) at 2700 days (see Figures 6(c) and $10(\mathrm{~d})$ ). For the last simulation, the damage shape is

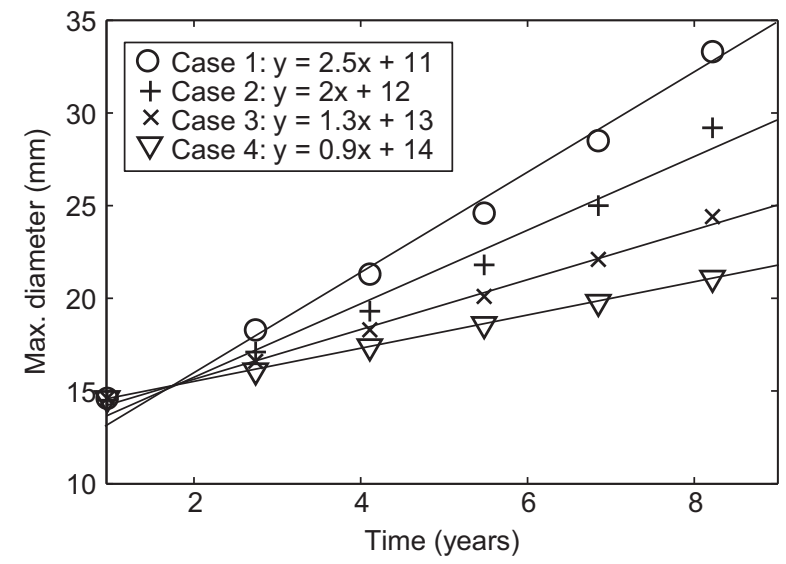

Figure 8. Expansion rates of the lesion in simulation Cases (1)-(4) as the maximum diameter of the lesion versus time and their best linear fit $\left(K_{g}=0.05\right)$. 


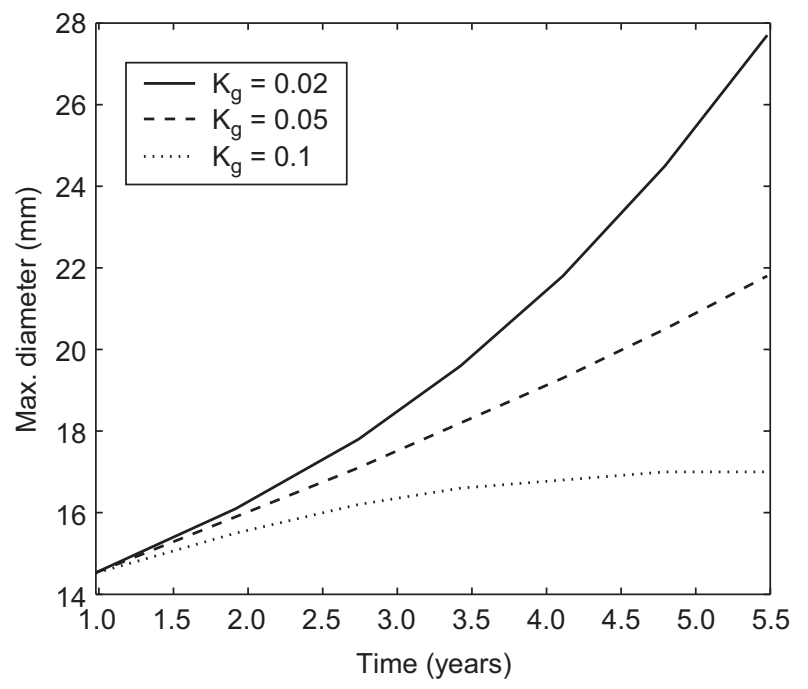

Figure 9. Expansion rates of the lesion in simulation Case (2) with different values of the kinetic parameter $\left(K_{g}=0.02,0.05\right.$ and 0.1).

initially the same as in Case (3) (Figure 11(a)), but elastin is degraded further with the 'stretch-induced' elastin damage. The simulation shows that the affected area gradually increases with stretch-induced damage (Figure 11(b)) and,
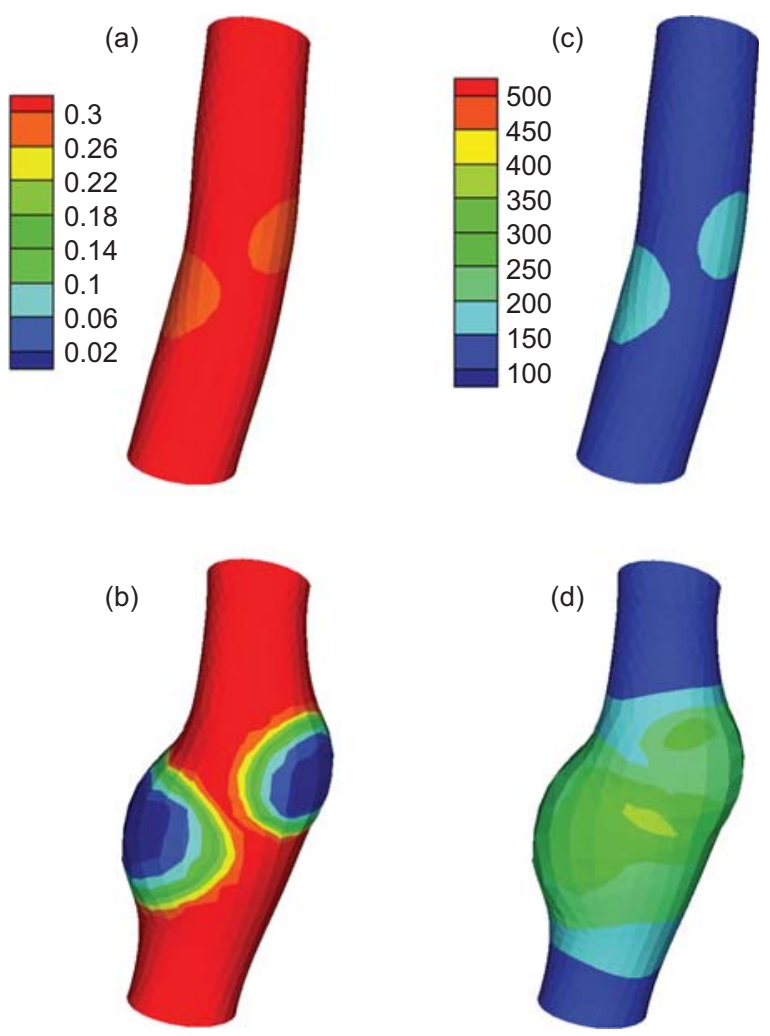

Figure 10. Areal mass density of elastin $\left(\mathrm{kg} / \mathrm{m}^{2}\right)$ for the case of 'time-dependent' degradation after 50 and 2700 days (a, b) and the corresponding maximum principal stress distribution (c, d) $(k P a)$.

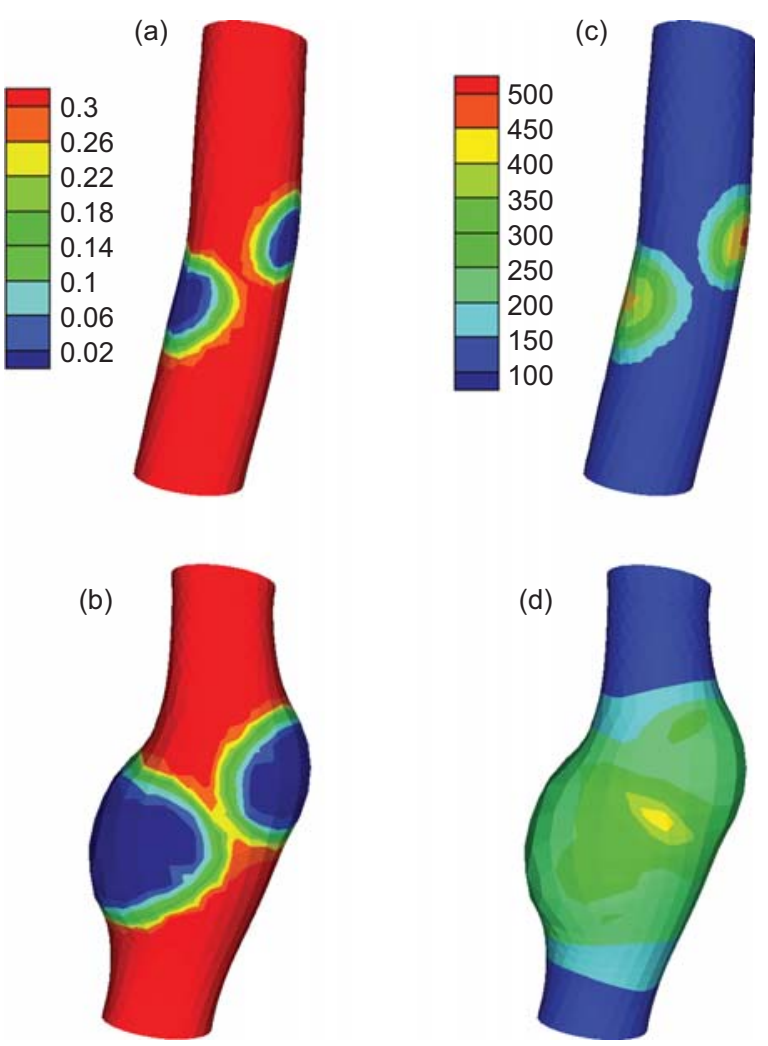

Figure 11. Areal mass density of elastin $\left(\mathrm{kg} / \mathrm{m}^{2}\right)$ for the case of 'stretch-induced' degradation after 50 and 2700 days (a, b) and the corresponding maximum principal stress distribution (c, d) $(k P a)$.

hence, the principal stress increases to a level higher than Case (3) at 2700 days without the stretch-induced damage (see Figures 6(c) and 11(d)).

\section{Discussion}

In this work, an FEM model of vascular adaptation is presented and applied to model the enlargement of an AAA without considering thrombus formation. We used a membrane model of arterial wall. Although a membrane model has some limitations, it is still preferable in many G\&R simulations (Gleason et al. 2004; Baek et al. 2006; Watton and Hill 2009). Watton and Hill (2009) suggested that the ratio of thickness of the wall to the diameter of the aneurysm decreases as an AAA enlarges and also the remodelling process tends to naturally maintain a uniform strain (or stress) field through the thickness. Hence, a membrane model suits to model the deformation of the abdominal aorta and the development of an aneurysm at physiological pressure. Moreover, a full 3D model of vascular adaptation needs more information about the variation of constituent properties through the thickness and their evolution during the vascular adaptation. 
Following Watton et al. (2004) and Watton and Hill (2009), elastin degradation was assumed to initiate our G\&R simulations and a similar form was used for spatial and temporal distribution of degradation. We considered, however, multiple spatial functions for elastin degradation which resulted in a variety of aneurysm shapes with different stress distributions and growth rates indicating the potential clinical application of computational simulation of G\&R with realistic geometries. Consistent with Watton and Hill (2009), it appears that collagen production tends to compensate for the loss of elastin. The computations suggest, however, that as a lesion evolves into a more complex shape, stress may be elevated at a location which was not necessarily a damage site (see Figure 6). In Cases 1 and 2, stress initially increased at locations where damage was introduced. Later, in the course of enlargement, the convex side of both aneurysms became the region of maximum stress leading to more dilation on this side. The results suggest that the location and the geometry of the lesion can influence AAA enlargement and rupture. For an AAA in vivo, however, the effect of geometry may be through its influence on both haemodynamics (e.g. wall shear stress; Hoi et al. 2004) and wall stress distribution (Vorp et al. 1998; Doyle et al. 2009). Asymmetric flow patterns have been associated with the formation of aneurysm in lower limb amputees via asymmetric distribution of wall shear stress (Vollmar et al. 1989; Paes et al. 1990; Naschitz and Lenger 2008). As expected, in our simulations, damage shapes which were more dispersed resulted in more dilation than localised damage shapes (see Figure 7). The 'time dependent' simulation for Case (3) did not result in a substantial difference in the aneurysm shape, but different time constants showed significant effects on the expansion rate (Figure 12). Although we used simple spatial and temporal functions for elastin degradation,

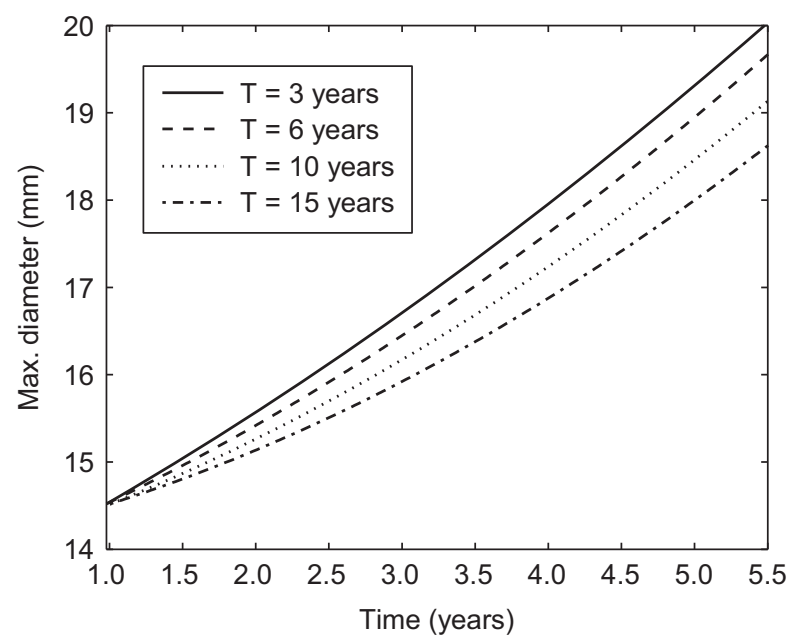

Figure 12. Expansion rates of the lesion in the 'time-dependent' simulation case associated with different time constants $(T=3,6,10$ and 15 years). elastin degradation during AAA growth involves multiple biological and mechanical parameters. It has been suggested that in AAAs, elastin degradation is due to the proteolytic activity which may have several causes including abnormal distribution of wall shear stress (Miller 2002; Hoshina et al. 2003; Sho et al. 2004), circumferential stress (Humphrey 2002), influx of inflammatory cells (Choke et al. 2005; Pearce and Shively 2006; Shimizu et al. 2006; Middleton et al. 2007) and the formation of the intraluminal thrombus layer (Vorp et al. 2001; Fontaine et al. 2002; Vorp and Vande Geest 2005). In our following study (Sheidaei et al. 2010), the current model is coupled with haemodynamic simulation in an iterative manner, and the effects of wall shear stress on the elastin degradation and, hence, on the aneurysm growth are investigated.

In our final simulation, we assumed that further degradation of elastin is induced by the stretch of elastin. Although it is not clear how much mechanical factors influence elastin degradation during the aneurysmal growth, previous ex vivo studies show that arterial elastin fails under uniaxial stretch (Gosline et al. 2002; Lillie and Gosline 2007). Then, it might not be unreasonable to hypothesise that the over-stretch of elastin accelerates elastin degradation. Previously, Wulandana and Robertson (2005) presented a model of a cerebral arterial tissue that accounts for a stretch-dependent failure mechanism of elastin. Recently, Li and Robertson (2009) have utilised a strain-induced damage model in a more structurally complicated model for balloon angioplasty.

We assumed that the amount of SM reduced proportionally with the initial elastin damage in our model. The results do not indicate a significant role of SM on AAA progression possibly because we did not incorporate direct effects of SM loss on the extracellular matrix turnover. However, there are increasing evidence on the critical role of vascular SM cells in the aneurysm pathobiology through their activity and quantity (Curci 2009). Vascular SM cells are capable of producing high levels of matrix degrading enzymes in AAAs (Patel et al. 1996; Crowther et al. 2000) as well as their inhibitors preventing the degeneration (Allaire et al. 1998). Stretch/stress-induced synthesis of extracellular matrix by SM cells has also been documented (Sumpio et al. 1988; O'Callaghan and William 2000). Apparently, the imbalance between proteolytic and synthetic activities of SM cells contributes to the structural deterioration of arterial wall. In addition, advanced stages of AAA have been associated with marked apoptosis of SM cells (López-Candales et al. 1997; Thompson et al. 1997; Zhang et al. 2003) which can exacerbate the weakening process. We need, however, more data to build a better model and predict the progressive weakening of the wall structure and its failure.

The half-life of collagen in the arterial wall is reported to be 60-70 days in normal conditions (Humphrey 2002), 
which can be reduced to 17 days in pathological conditions (Nissen et al. 1978). Watton and Hill (2009) showed that reducing the half-life time nonlinearly increased the expansion rate. Our preliminary results also showed that expansion rate is inversely related to half-life time. We chose the half-life time to be about 35 days in our simulations. Our results demonstrated that the kinetic parameter, $K_{g}$, has a key effect on the expansion rate, (Figure 9) consistent with other studies (Baek et al. 2005; Baek et al. 2006). Nonetheless, the collagen half-life as well as the kinetic parameters was assumed to be fixed during the evolution of aneurysm in our model, whereas in an actual AAA, these parameters may change during aneurysm growth due to pathological changes. Dynamic changes of turnover parameters are multifaceted and complex and, hence, more studies are required to quantify these pathological changes in aneurysm growth. Also, individuals may have different degrees of mechano-sensitivity and stress-mediated turnover of collagen depending of their physiological and pathological conditions. In fact, findings on the collagen content variation during AAA growth are highly variable, e.g. decreased (Sumner et al. 1970), increased (Ghorpade and Baxter 1996) and no change (Rizzo et al. 1989). The simulation showed that $K_{g}=0.05$ provides almost linear growth for all damage shapes (Figure 8) and, especially, for Cases (1) and (3), growth rates were within the range observed for small aneurysms (Nevitt et al. 1989; Baxter et al. 2008). With $K_{g} \leq 0.05$, the lesion enlarged continuously implying that the stressmediated collagen turnover was not enough to return the stress back to the homeostatic level, although it reduced the local damage-induced stress (Figure 9).

Results from the current model represent the early stage of aneurysms. As AAAs grow, other factors such as intraluminal thrombus and perivascular boundary conditions should also be taken into account for the model. Intraluminal thrombus not only has a direct mechanical effect (Wang et al. 2006) but also changes the chemomechanical environment and influences the strength of the lesion and its progression (Vorp et al. 2001; Taylor and Humphrey 2009).

Remodelling of the constituents and the mechanism involved in their deposition can have a great impact on the mechanical properties of the evolved tissue. Computational models of collagen remodelling assumed that either the principal strains (Boerboom et al. 2003; Driessen et al. 2004, 2008) or stresses (Baek et al. 2006; Hariton et al. 2007) govern the orientation of collagen fibres. With regard to AAA, marked increase of anisotropy was found in the diseased tissue (Vande Geest et al. 2006), suggesting structural changes in aneurysm formation. Watton et al. (2004) assumed fixed fibre directions for collagen fibres in their AAA G\&R simulations. In our model, collagen aligned towards existing fibre directions. With newly deposited collagen during enlargement, anisotropy increased in the circumferential direction, which is consistent with that in Vande Geest et al. (2006). In addition, we considered the mechanical property of newly synthesised collagen fibres to be the same (Humphrey 1999; Gleason et al. 2004; Baek et al. 2006; Baek et al. 2007). Elastin degradation alone does not conduce to rupture in normal vessels (Dobrin et al. 1984). Impaired collagen networking and its microstructural defects have been associated with advanced AAAs despite an increase in the collagen concentration (Lindeman et al. 2010). Therefore, in addition to alteration of fibre distribution, it might also be desirable to account for changes of collagen type, cross-linking and fibre thickness in the model (Driessen et al. 2008). A future extension of the current model can be towards accounting for alteration of collagen structure during the AAA progression and different mechanical properties of the newly synthesised collagen fibres.

Prescribing the in vivo material and geometric parameters that satisfy the balance of momentum and homeostatic conditions is a major challenge for patientspecific G\&R simulations. In the current work, we approximated the thickness by the Laplace relation, $\sigma_{h}=\operatorname{Pr} / h$, where $r$ is the first principal radius of curvature. For a more complex geometry, however, there is a need for developing numerical techniques to identify the spatial distribution of parameters and integrating them with the developed vascular adaptation model.

Previous computational studies of vascular adaptation have considered mostly axisymmetric and simple geometries, while clinical application of the G\&R simulation demands patient-specific anatomical information. Watton et al. (2004) simulated an asymmetric aneurysm by assuming an axisymmetric degradation and considering an effective pressure (instead of constant pressure) which resembles the contact with spine. Although varying the effective pressure resulted in an asymmetric aneurysm growth, their initial configuration was still axisymmetric. Recently Kuhl et al. (2007) simulated stress-induced growth of a medical image-based model of human aorta based on the concept of incompatible growth combined with open system thermodynamics. They simulated the effect of balloon or stent exposure in atherosclerotic patients by a high internal pressure locally applied to the wall. Their work, however, was based on a singleconstituent approach and did not consider G\&R of multiple constituents. On the other hand, many studies have used patient-specific geometries for estimating accurate stress levels and the rupture risk of aneurysms without a G\&R mechanism (Raghavan and Vorp 2000; Fillinger et al. 2002, 2003; Speelman et al. 2007). However, Vorp and Vande Geest (2005) suggested that 'despite recent reports, it should be noted that evaluation of rupture potential based on only one of these parameters - stress or strength - is not sufficient because a region of the AAA wall that is under 
elevated wall stress may also have a higher wall strength'. We submit that by coupling a G\&R model with a patientspecific geometric model, a computational simulation can provide information about both stress and structural strength during aneurysm growth and, hence, better prediction of the rupture can be acquired. Therefore, the computational model presented here will provide a useful foundation towards a patient-specific modelling of AAAs.

\section{Acknowledgement}

The authors thank Prof. Jay D. Humphrey at Texas A\&M University for his invaluable suggestions.

\section{References}

Ailawadi G, Moehle CW, Pei H, Walton SP, Yang Z, Kron IL, Lau CL, Owens GK. 2009. Smooth muscle phenotypic modulation is an early event in aortic aneurysms. J Thorac Cardiovasc Surg. 138(6):1392-1399.

Allaire E, Forough R, Clowes M. 1998. Local overexpression of TIMP-1 prevents aortic aneurysm degeneration and rupture in a rat model. J Clin Invest. 102(7):1413-1420.

Baek S, Rajagopal KR, Humphrey JD. 2005. Competition between radial expansion and thickening in the enlargement of an intracranial saccular aneurysm. J Elast. 80:13-31.

Baek S, Rajagopal KR, Humphrey JD. 2006. A theoretical model of enlarging intracranial fusiform aneurysms. J Biomech Eng. 128(1):142-149.

Baek S, Valentín A, Humphrey JD. 2007. Biochemomechanics of cerebral vasospasm and its resolution: II. Constitutive relations and model simulations. Ann Biomed Eng. 35(9):1498-1509.

Baxter BT, Terrin MC, Dalman RL. 2008. Medical management of small abdominal aortic aneurysms. Circulation. 117(14):1883-1889.

Boerboom RA, Driessen NJB, Bouten CVC, Huyghe FPTB JM. 2003. Finite element model of mechanically induced collagen fiber synthesis and degradation in the aortic valve. Ann Biomed Eng. 31(9):1040-1053.

Burton AC. 1954. Relation of structure to function of the tissues of the wall of blood vessels. Physiol Rev. 34(4):619-642.

Choke E, Cockerill G, Wilson WRW, Sayed S, Dawson J, Loftus I, Thompson MM. 2005. A review of biological factors implicated in abdominal aortic aneurysm aupture. Eur J Vasc Endovasc Surg. 30(3):227-244.

Crowther M, Goodall S, Jones JL, Bell PR, Thompson MM. 2000. Increased matrix metalloproteinase 2 expression in vascular smooth muscle cells cultured from abdominal aortic aneurysms. J Vasc Surg. 32(3):575-583.

Curci JA. 2009. Digging in the "Soil" of the aorta to understand the growth of abdominal aortic aneurysms. Vascular. 17:S21-S29.

Dobrin PB, Baker WH, Gley WC. 1984. Elastolytic and collagenolytic studies of arteries. Arch Surg. 119(4):405-409.

Doyle BJ, Callanan A, Burke PE, Grace PA, Walsh MT, Vorp DA, McGloughlin TM. 2009. Vessel asymmetry as an additional diagnostic tool in the assessment of abdominal aortic aneurysms. J Vasc Surg. 49(2):443-454.

Driessen NJB, Wilson W, Bouten CVC, Baaijens FPT. 2004. A computational model for collagen fibre remodelling in the arterial wall. J Theor Biol. 226(1):53-64.

Driessen NJB, Cox MAJ, Bouten CVC, Baaijens FPT. 2008. Remodelling of the angular collagen fiber distribution in cardiovascular tissues. Biomech Model Mechanobiol. 7(2):93-103.
Driss AG, Benessiano J, Poitevin P, Levy BI, Michael JB. 1997. Arterial expansive remodeling induced by high flow rates. Am J Physiol. 272(2):H851-H858.

Figueroa CA, Baek S, Taylor CA, Humphrey JD. 2009. A computational framework for fluid-solid-growth modeling in cardiovascular simulations. Comput Methods Appl Mech Eng. 198:3583-3602.

Fillinger MF, Raghavan ML, Marra SP, Cronenwell JL, Kennedy FE. 2002. In vivo analysis of mechanical wall stress and abdominal aortic aneurysm rupture risk. J Vasc Surg. 36(3): 589-597.

Fillinger MF, Marra SP, Raghavan ML, Kennedy FE. 2003. Prediction of rupture risk in abdominal aortic aneurysm during observation: wall stress versus diameter. J Vasc Surg. 37(4):724-732.

Fontaine V, Jacob MP, Houard X, Rossignol P, Plissonnier D, Angles-Cano E, Michel JB. 2002. Involvement of the mural thrombus as a site of protease release and activation in human aortic aneurysms. Am J Pathol. 161(5):1701-1710.

Ghorpade A, Baxter BT. 1996. Biochemistry and molecular regulation of matrix macromolecules in abdominal aortic aneurysms. Ann N Y Acad Sci. 800:138-150.

Gleason RL, Taber LA, Humphrey JD. 2004. A 2-D model of flowinduced alterations in the geometry, structure, and properties of carotid arteries. J Biomech Eng. 126(3):371-381.

Gosline J, Lillie M, Carrington E, Guerette P, Ortlepp C, Savage K. 2002. Elastic proteins: biological roles and mechanical properties. Philos Trans R Soc Lond B Biol Sci. 357(1418): $121-132$.

Hariton I, deBotton G, Gasser TC, Holzapfel GA. 2007. Stressmodulated collagen fiber remodeling in a human carotid bifurcation. J Theor Biol. 248(3):460-470.

He CM, Roach MR. 1994. The composition and mechanical properties of abdominal aortic aneurysms. J Vasc Surg. 20(1):6-13.

Hoi Y, Meng H, Woodward SH, Bendok BR, Hanel RA, Guterman LR, Hopkins LN. 2004. Effects of arterial geometry on aneurysm growth: three-dimensional computational fluid dynamics study. J Neurosurg. 101(4):676-681.

Holzapfel GA, Gasser TG, Ogden RW. 2000. A new constitutive framework for arterial wall mechanics and a comparative study of material models. J Elast. 61:1-48.

Hoshina K, Sho E, Sho M, Nakahashi TK, Dalman RL. 2003. Wall shear stress and strain modulate experimental aneurysm cellularity. J Vasc Surg. 37(5):1067-1074.

Humphrey JD. 1999. Remodeling of collagenous tissue at fixed lengths. J Biomech Eng. 121:591-597.

Humphrey JD. 2002. Cardiovascular solid mechanics: cells, tissues, and organs. New York: Springer-Verlag.

Humphrey JD, Na S. 2002. Elastodynamics and arterial wall stress. Ann Biomed Eng. 30(4):509-523.

Humphrey JD, Rajagopal KR. 2002. A constrained mixture model for growth and remodeling of soft tissues. Math Models Methods Appl Sci. 12(3):407-430.

Humphrey JD, Eberth JF, Dye WW, Gleason RL. 2009. Fundamental role of axial stress in compensatory adaptations by arteries. J Biomech. 42(1):1-8.

Jackson ZS, Dajnoweiec D, Gotlieb AI, Langille BL. 2005. Partial off-loading of longitudinal tension induces arterial tortuosity. Arterioscler Thromb Vasc Biol. 25(5):957-962.

Karnik SK, Brooke BS, Bayes-Genis A, Sorensen L, Wythe JD, Schwartz RS, Keating MT, Li DY. 2003. A critical role for elastin signaling in vascular morphogenesis and disease. Development. 130(2):411-423. 
Kroon M, Holzapfel GA. 2007. A model for saccular cerebral aneurysm growth by collagen fibre remodelling. J Theor Biol. 247(4):775-787.

Kroon M, Holzapfel GA. 2009. A theoretical model for fibroblast-controlled growth of saccular cerebral aneurysms. J Theor Biol. 257(1):73-83.

Kuhl E, Maas R, Himpel G, Menzel A. 2007. Computational modeling of arterial wall growth. Attempts towards patientspecific simulations based on computer tomography. Biomech Model Mechanobiol. 6(5):321-331.

Kyriacou SK, Schwab C, Humphrey JD. 1996. Finite element analysis of nonlinear orthotropic hyperelastic membranes. Comput Mech. 18(4):269-278.

Li D, Robertson AN. 2009. A structural multi-mechanism damage model for cerebral arterial tissue. J Biomech Eng. 131(10):101013.

Li DY, Brooke B, Davis EC, Mecham RP, Boak LKSBB, Eichwald E, Keating MT. 1998. Elastin is an essential determinant of arterial morphogenesis. Nature. 393(6682): 276-280.

Lillie MA, Gosline JM. 2007. Mechanical properties of elastin along the thoracic aorta in the pig. J Biomech. 40(10):2214-2221.

Lindeman JHN, Ashcroft BA, Beenakker JM, vanEs M, Koekkoek NBR, Prins FA, Tielemans JF, Abdul-Hussien H, Bank RA, Oosterkamp TH. 2010. Distinct defects in collagen microarchitecture underlie vessel-wall failure in advanced abdominal aneurysms and aneurysms in Marfan syndrome. Proc Natl Acad Sci USA. 107(2):862-865.

López-Candales A, Holmes DR, Liao S, Scott MJ, Wickline SA, Thompson RW. 1997. Decreased vascular smooth muscle cell density in medial degeneration of human abdominal aortic aneurysms. Am J Pathol. 150(3):993-1007.

Menzel A. 2005. Modelling of anisotropic growth in biological tissues. A new approach and computational aspects. Biomech Model Mechanobiol. 3(3):147-171.

Middleton RK, Lloyd GM, Bown MJ, Cooper NJ, London NJ, Sayers RD. 2007. The pro-inflammatory and chemotactic cytokine microenvironment of the abdominal aortic aneurysm wall: a protein array study. J Vasc Surg. 45(3):574-580.

Miller FJ. 2002. Aortic aneurysms: it's all about the stress. Arterioscler Thromb Vasc Biol. 22(12):1948-1949.

Mulvany MJ. 1992. Vascular growth in hypertension. J Cardiovasc Pharmacol. 20:S11-S17.

Naschitz JE, Lenger R. 2008. Why traumatic leg amputees are at increased risk for cardiovascular diseases. QJM. 101(4): 251-259.

Nevitt MP, Ballard DJ, Hallett JW. 1989. Prognosis of abdominal aortic aneurysms. A population-based study. N Engl J Med. 321(15):1009-1014.

Nissen R, Cardinale GJ, Udenfriend S. 1978. Increased turnover of arterial collagen in hypertensive rats. Proc Natl Acad Sci. 75(1):451-453.

O'Callaghan CJ, William B. 2000. Mechanical strain induced extracellular matrix production by human vascular smooth muscle cells. Hypertension. 36(3):319-324.

Paes EH, Vollmar JF, Pauschinger P, Mutschler W, Henze E, Friesch A. 1990. Late vascular damage after unilateral leg amputation. Z Unfallchir Versicherungsmed. 83(4):227-236.

Park HC, Youn S. 1998. Finite element analysis and constitutive modelling of anisotropic nonlinear hyperelastic bodies with convected frames. Comput Methods Appl Mech Eng. 151(3-4):605-618.

Patel MI, Melrose J, Ghosh P, Appleberg M. 1996. Increased synthesis of matrix metalloproteinases by aortic smooth muscle cells is implicated in the etiopathogenesis of abdominal aortic aneurysms. J Vasc Surg. 24(1):82-92.

Pearce WH, Shively VP. 2006. Abdominal aortic aneurysm as a complex multifactorial disease: interaction of polymorphisms of inflammatory genes, features of autoimmunity, and current status of MMPs. Ann N Y Acad Sci. 1085:117-132.

Powell JT. 2002. Abdominal aortic aneurysm. An introduction to vascular biology. 2 ed. Cambridge: Cambridge University Press.

Press W, Teukolsky S, Vetterling W, Flannery B. 1992. Numerical Recipes in C. 2nd ed. Cambridge: Cambridge University Press.

Raghavan ML, Vorp DA. 2000. Toward a biomechanical tool to evaluate rupture potential of abdominal aortic aneurysm: identification of a finite strain constitutive model and evaluation of its applicability. J Biomech. 33(4):475-482.

Rizzo RJ, McCarthy WJ, Dixit SN, Lilly MP, Shively VP, Flinn WR, Yao JST. 1989. Collagen types and matrix protein content in human abdominal aortic aneurysms. J Vasc Surg. 10(4):365-373.

Sheidaei A, Hunley SC, Zeinali-Davarani S, Raguin LG, Baek S. 2010. Simulation of abdominal aortic aneurysm growth with updating hemodynamic loads using a realistic geometry. Med Eng Phys. 33(1):80-88.

Shimizu K, Mitchell RN, Libby P. 2006. Inflammation and cellular immune responses in abdominal aortic aneurysms. Arterioscler Thromb Vasc Biol. 26(5):987-994.

Sho E, Sho M, Hoshina K, Kimura H, Nakahashi TK, Dalman RL. 2004. Hemodynamic forces regulate mural macrophage infiltration in experimental aortic aneurysms. Exp Mol Pathol. 76(2): 108-116.

Speelman L, Bohra A, Boosman EMH, Schurink GHW, van deVosse FN, Makaroun MS, Vorp DA. 2007. Effects of wall calcifications in patient-specific wall stress analyses of abdominal aortic aneurysms. J Biomech Eng. 129(1): 105-109.

Sumner DS, Hokanson DE, Strandness DE. 1970. Stress-strain characteristics and collagen-elastin content of abdominal aortic aneurysms. Surg Gynecol Obstet. 130(3):459-466.

Sumpio BE, Banes AJ, Link WG, Johnson Jr. G. 1988. Enhanced collagen production by smooth muscle cells during repetitive mechanical stretching. Arch Surg. 123(10):1233-1236.

Taylor CA, Humphrey JD. 2009. Open problems in computational vascular biomechanics: hemodynamics and arterial wall mechanics. Comput Methods Appl Mech Eng. 198(45-46): 3514-3523.

Thompson RW, Liao SX, Curci JA. 1997. Vascular smooth muscle cell apoptosis in abdominal aortic aneurysms. Coron Artery Dis. 8(10):623-631.

Truesdell C, Noll W. 1965. The non-linear field theories of mechanics. In: Flugge S, editor. Handbuch der Physik. vol. III/3. Berlin: Springer.

Valentín A, Cardamone L, Baek S, Humphrey JD. 2009. Complementary vasoactivity and matrix remodeling in arterial adaptations to altered flow and pressure. J R Soc Interface. 6(32):293-306.

Vande Geest JP, Sacks MS, Vorp DA. 2006. The effects of aneurysm on biaxial mechanical behavior of human abdominal aorta. J Biomech. 39(7):1324-1334.

Vollmar JF, Pauschinger P, Paes E, Henze E, Friesch A. 1989. Aortic aneurysms as late sequelae of above-knee amputation. Lancet. 334(8667):834-835.

Vorp DA, Vande Geest JP. 2005. Biomechanical determinants of abdominal aortic aneurysm rupture. Arterioscler Thromb Vasc Biol. 25(8):1558-1566. 
Vorp DA, Raghavan ML, Webster MW. 1998. Stress distribution in abdominal aortic aneurysm: influence of diameter and asymmetry. J Vasc Surg. 27(4):632-639.

Vorp DA, Lee PC, Wang DHJ, Makaroun MS, Nemoto EM, Ogawa S, Webster MW. 2001. Association of intraluminal thrombus in abdominal aortic aneurysm with local hypoxia and wall weakening. J Vasc Surg. 34(2):291-299.

Wang C, Garcia M, Lu X, Lanir Y, Kassab GS. 2006. Threedimensional mechanical properties of porcine coronary arteries: a validated two-layer model. Am J Physiol Heart Circ Physiol. 291(3):1200-1209.

Watton PN, Hill NA. 2009. Evolving mechanical properties of a model of abdominal aortic aneurysm. Biomech Model Mechanobiol. 8(1):25-42.
Watton PN, Hill NA, Heil M. 2004. A mathematical model for the growth of the abdominal aortic aneurysm. Biomech Model Mechanobiol. 3(2):98-113.

Wulandana R, Robertson AM. 2005. An inelastic multimechanism constitutive equation for cerebral aretrial tissue. Biomech Model Mechanobiol. 4(4):235-248.

Zhang J, Schmidt J, Ryschich E, Schumacher H, Allenberg JR. 2003. Increased apoptosis and decreased density of medial smooth muscle cells in human abdominal aortic aneurysms. Chin Med. J. 116(10):1549-1552.

Zulliger MA, Rachev A, Stergiopulos N. 2004. A constitutive formulation of arterial mechanics including vascular smooth muscle tone. Am J Physiol Heart Circ Physiol. 287(3):H1335-H1343. 